\title{
List of Charts and Graphs
}

Chart 1 A Comparison of the Frequency of Motifs within the Entire Assemblage - 185

Chart 2 A Comparison of the Frequency of the Motifs on the Architectural Elements in the Entire Assemblage - 186

Chart 3 Frequency of Geometric Motifs According to Architectural Element Types - 190

Chart 4 Types of Architectural Elements in the Entire Assemblage - 190

Chart 5 Frequency of Rosettes According to Architectural Element Types - 191

Chart 6 Types of Architectural Elements in the Entire Assemblage - 192

Chart 7 Frequency of Floral Motifs According to Architectural Element Types - 193

Chart 8 Types of Architectural Elements in the Entire Assemblage - 193

Chart 9 Frequency of Faunal Motifs According to Architectural Element Types - 195

Chart 10 Types of Architectural Elements in the Entire Assemblage - 195

Chart 11 Frequency of Architectural Motifs According to Architectural Element Types - 196

Chart 12 Types of Architectural Elements in the Entire Assemblage - 196

Chart 13 Frequency of the Object-Type Motif According to Architectural Element Types - 197

Chart 14 Types of Architectural Elements in the Entire Assemblage - 198

Graph 15 Distribution of Exclusively Geometric Compositions Compared to the Total Number of Elements in the Assemblage $-\mathbf{2 1 0}$

Graph 16 Distribution of Hybrid Compositions - 212

Chart and The Frequencies of decorated: Floor Tiles (FT), Bases and Podia (BP), Doorjambs

Graph 17 (D)), Lintels (LT), Columns (CL), Capitals (CP), Cornices (CR) and Arches (AR) -212 
